\title{
15. THE Me VARIABLES AND GALACTIC STRUCTURE
}

\author{
M. W. Feast \\ Radcliffe Observatory
}

Recently a program of radial velocity determinations for 114 southern longperiod variables has been completed. Full details of these results and an analysis of them are being published elsewhere (Feast 1963). Below are summarized some of the results which seem of particular interest for problems of galactic structure.

Three hundred and fifty-three Me variables were divided into seven groups according to period. The radial velocities were corrected for basic solar motion and analysedfor :

1. The systematic motion $U, V, W$ in galactic coordinates.

2. The constants of the velocity ellipsoid $h, k, l$.

3. Differential galactic rotation.

The $K$ term is zero. There is no deviation of the anti-apex of the systematic motion from the direction of galactic rotation. On Kerr's model of the Galaxy we would expect the older stars such as the Me variables to show a mean inward motion of $7 \mathrm{~km} / \mathrm{sec}$ with respect to the younger stars and the gas. Instead a net outward motion of $3 \cdot 8 \pm 3 \cdot 6$ (s.e.) $\mathrm{km} / \mathrm{sec}$ is found.

The observed variation with period of the systematic motion, $V$, in the direction of galactic rotation together with previous work on the three $\mathrm{Me}$ variables in the globular cluster 47 Tuc (Feast and Thackeray 1960) may be used to infer ages of 1 or $2 \times 10^{10}$ years, masses of about the solar mass and chemical composition with slight metal deficiencies (about a factor 4 compared to the Sun) for the shorter period stars ( $\sim 175^{\mathrm{d}}$ periods). For the longer period stars $\left(\sim 450^{\mathrm{d}}\right.$ periods) ages of about $5 \times 10^{8}$ years, masses of about $2 \cdot 5$ solar masses, and chemical compositions rather similar to the Sun's may be estimated. The bulk of the Me variables are associated with the disk and intermediate Population II and at maximum light are probably the brightest stars of this population type.

The anomalous motions of the Me variables with periods less than 149 days suggest that these stars are pulsating in the first overtone and are related to the longer period variables in the same way that the $c$ type $R R$. Lyrae stars are related to the ab types. The ratio of the pulsation constants $Q_{0} / Q_{1}(2 \cdot 4)$ and the value of $Q_{0}(0 \cdot 056)$ derived are in accord with this hypothesis.

The mean space density of the Me variables is decreasing outwards as $R^{2 \cdot 9}$. This gradient agrees with the value found by Oort for the general field of stars at large distances from the galactic plane. The density gradient becomes less steep with increasing $R$.

The value of $h / l$ - the ratio of the axes of the velocity ellipsoid in the direction of the poles and in the plane radial from the centre - is $0 \cdot 77 \pm 0 \cdot 14$ (s.e.). Younger groups of stars yield values near $\mathbf{0 . 5}$ whilst Jeans's theorem requires a ratio of unity for the steady state. If Jeans's theorem holds for the Galaxy we may conclude that the Me variables are sufficiently old to have approached more closely to the steady state than other groups of stars such as the main sequence A types. However, recent 
work has indicated the possibility that the galactic potential field is of such a form that it may admit of a third integral of the equations of motion. Should this be so the ratio $h / l$ need not be unity even in the steady state.

Evidence for differential galactic rotation is found. A weighted mean value of the Oort constant $A$ is $+7 \cdot 8 \pm 3 \cdot 4$ (s.e.) $\mathrm{km} / \mathrm{sec} . \mathrm{kpc}$. Whilst this is considerably smaller than the value appropriate to extreme Population I objects, it agrees well with the value that can be predicted from the velocity ellipsoids and systematic motions $(5 \mathrm{~km} / \mathrm{sec} . \mathrm{kpc})$.

Finally, differential galactic rotation for the Se variables on assuming them to be extreme Population I objects yields a visual absolute magnitude at maximum of $-3^{\mathrm{m}} 4 \pm 0 \mathrm{~m} 8$ (s.e.). This is much brighter than the value of -1.956 recently found from statistical parallaxes.

\section{References}

FEAST, M. W. (1963).-M.N. 125: 367-415.

Feast, M. W., and Thackeray, A. D. (1960).-M.N. $120: 463-82$.

\section{Discussion}

Landi Dessy: Do you measure emission or absorption lines?

Feast: Both, if possible. Eighty-six of the 114 have absorption as well as emission lines.

Landi Dessy: Do you always measure them at maximum?

Feast : Yes, but we have followed a few to lower brightness.

\section{GALACTIC STRUCTURE AND ROTATION FROM CEPHEIDS}

\section{R. P. Kraft and M. Schmidt}

\section{Mount Wilson and Palomar Observatories}

Internally consistent distances to classical cepheids can be derived with considerable accuracy because they satisfy period-luminosity and period-colour relations. Studies of five cepheids that are members of galactic clusters have led to a revised period-colour relation and a new zero point for the period-luminosity relation. Distances have been computed for all cepheids given in recently published photoelectric surveys. The material can be regarded as complete to a distance of about $1500 \mathrm{pc}$.

The distribution of cepheids of all periods shows that within 1500 pc the vast majority of cepheids are on the side of the Sun toward the centre of the Galaxy. The only clear indication of structure in the distribution is seen toward $l \mathrm{II}=290^{\circ}$. This is probably the inner edge of a spiral arm in the Carina region, the central part of which may be lost owing to high obscuration at lower longitudes.

Long-period cepheids, with $M_{V} \leqslant-4 \cdot 3$, show a distribution quite similar to that exhibited by $\mathrm{OB}$ associations. Concentrations or spiral arms may be noted in Cyg and in the Per-Cas region. It is not clear how, or whether, these concentrations extend into the area $l \mathrm{II}=180$ to $360^{\circ}$. The Per-Cas arm seems to vanish in the direction of the anticentre. The $\mathrm{OB}$ associations are distributed roughly symmetrically about the Sun (within $1500 \mathrm{pc}$ ), in marked contrast with the asymmetry exhibited by cepheids of all periods. There are too few long-period cepheids within $1500 \mathrm{pc}$ to say anything about their distribution locally. 